Geometry $8 \mathcal{F}$ Topology

Volume 4 (2000) 451-456

Published: 26 November 2000

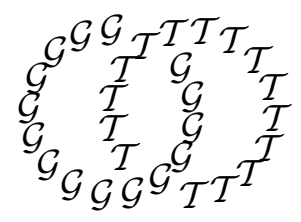

\title{
Diffeomorphisms, symplectic forms and Kodaira fibrations
}

\author{
Claude LeBrun \\ Department of Mathematics, SUNY at Stony Brook \\ Stony Brook, NY 11794-3651, USA \\ Email: claude@math.sunysb.edu
}

\begin{abstract}
As was recently pointed out by McMullen and Taubes [7], there are 4-manifolds for which the diffeomorphism group does not act transitively on the deformation classes of orientation-compatible symplectic structures. This note points out some other 4 -manifolds with this property which arise as the orientationreversed versions of certain complex surfaces constructed by Kodaira [3]. While this construction is arguably simpler than that of McMullen and Taubes, its simplicity comes at a price: the examples exhibited herein all have large fundamental groups.
\end{abstract}

AMS Classification numbers Primary: 53D35

Secondary: 14J29, 57R57

Keywords: Symplectic manifold, complex surface, Seiberg-Witten invariants

Proposed: Ronald Stern

Seconded: Yasha Eliashberg, Ronald Fintushel
Received: 11 June 2000

Accepted: 21 November 2000

Copyright Geometry and Topology 
Let $M$ be a smooth, compact oriented 4 -manifold. If $M$ admits an orientationcompatible symplectic form, meaning a closed 2 -form $\omega$ such that $\omega \wedge \omega$ is an orientation-compatible volume form, one might well ask whether the space of such forms is connected. In fact, it is not difficult to construct examples where the answer is negative. A more subtle question, however, is whether the group of orientation-preserving diffeomorphisms $M \rightarrow M$ acts transitively on the set of connected components of the orientation-compatible symplectic structures of $M$. As was recently pointed out by McMullen and Taubes [7], there are 4-manifolds $M$ for which this subtler question also has a negative answer. The purpose of the present note is to point out that many examples of this interesting phenomenon arise from certain complex surfaces with Kodaira fibrations.

A Kodaira fibration is by definition a holomorphic submersion $f: M \rightarrow B$ from a compact complex surface to a compact complex curve, with base $B$ and fiber $F_{z}=f^{-1}(z)$ both of genus $\geq 2$. (In $C^{\infty}$ terms, $f$ is thus a locally trivial fiber bundle, but nearby fibers of $f$ may well be non-isomorphic as complex curves.) One says that $M$ is a Kodaira-fibered surface if it admits such a fibration $f$. Now any Kodaira-fibered surface $M$ is algebraic, since $K_{M} \otimes f^{*} K_{B}^{\otimes \ell}$ is obviously positive for sufficiently large $\ell$. On the other hand, recall that a holomorphic map from a curve of lower genus to a curve of higher genus must be constant. ${ }^{1}$ If $f: M \rightarrow B$ is a Kodaira fibration, it follows that $M$ cannot contain any rational or elliptic curves, since composing $f$ with the inclusion would result in a constant map, and the curve would therefore be contained in a fiber of $f$; contradiction. The Kodaira-Enriques classification [2] therefore tells us that $M$ is a minimal surface of general type. In particular, the only non-trivial Seiberg-Witten invariants of the underlying oriented 4-manifold $M$ are [8] those associated with the canonical and anti-canonical classes of $M$. Any orientation-preserving self-diffeomorphism of $M$ must therefore preserve $\left\{ \pm c_{1}(M)\right\}$.

We have just seen that $M$ is of Kähler type, so let $\psi$ denote some Kähler form on $M$, and observe that $\psi$ is then of course a symplectic form compatible with the usual 'complex' orientation of $M$. Let $\varphi$ be any area form on $B$, compatible with its complex orientation, and, for sufficiently small $\varepsilon>0$, consider the closed 2-form

$$
\omega=\varepsilon \psi-f^{*} \varphi
$$

\footnotetext{
${ }^{1}$ Indeed, by Poincaré duality, a continuous map $h: X \rightarrow Y$ of non-zero degree between compact oriented manifolds of the same dimension must induce inclusions $h^{*}: H^{j}(Y, \mathbb{R}) \hookrightarrow H^{j}(X, \mathbb{R})$ for all $j$. Such a map $h$ therefore cannot exist whenever $b_{j}(X)<b_{j}(Y)$ for some $j$.
}

Geometry and Topology, Volume 4 (2000) 
Then

$$
\frac{\omega \wedge \omega}{\varepsilon}=-2\left(f^{*} \varphi\right) \wedge \psi+\varepsilon \psi \wedge \psi=\left(\varepsilon-\left\langle f^{*} \varphi, \psi\right\rangle\right) \psi \wedge \psi,
$$

where the inner product is taken with respect to the Kähler metric corresponding to $\psi$. Now $\left\langle f^{*} \varphi, \psi\right\rangle$ is a positive function, and, because $M$ is compact, therefore has a positive minimum. Thus, for a sufficiently small $\varepsilon>0, \omega \wedge \omega$ is a volume form compatible with the non-standard orientation of $M$; or, in other words, $\omega$ is a symplectic form for the reverse-oriented 4 -manifold $\bar{M}$. For related constructions of symplectic structures on fiber-bundles, cf [6].

If follows that $\bar{M}$ carries a unique deformation class of almost-complex structures compatible with $\omega$. One such almost-complex structure can be constructed by considering the (non-holomorphic) orthogonal decomposition

$$
T M=\operatorname{ker}\left(f_{*}\right) \oplus f^{*}(T B)
$$

induced by the given Kähler metric, and then reversing the sign of the complex structure on the 'horizontal' bundle $f^{*}(T B)$. The first Chern class of the resulting almost-complex structure is thus given by

$$
c_{1}(\bar{M}, \omega)=c_{1}(M)-4(1-\mathbf{g}) F,
$$

where $\mathbf{g}$ is the genus of $B$, and where $F$ now denotes the Poincaré dual of a fiber of $f$. For further discussion, cf $[4,5,9]$.

Of course, the product $B \times F$ of two complex curves of genus $\geq 2$ is certainly Kodaira fibered, but such a product also admits orientation-reversing diffeomorphisms, and so, in particular, has signature $\tau=0$. However, as was first observed by Kodaira [3], one can construct examples with $\tau>0$ by taking branched covers of products; $\mathrm{cf}[1,2]$.

Example Let $C$ be a compact complex curve of genus $k \geq 2$, and let $B_{1}$ be a curve of genus $\mathbf{g}_{1}=2 k-1$, obtained as an unbranched double cover of $C$. Let $\iota: B_{1} \rightarrow B_{1}$ be the associated non-trivial deck transformation, which is a free holomorphic involution of $B_{1}$. Let $p: B_{2} \rightarrow B_{1}$ be the unique unbranched cover of order $2^{4 k-2}$ with $p_{*}\left[\pi_{1}\left(B_{2}\right)\right]=\operatorname{ker}\left[\pi_{1}\left(B_{1}\right) \rightarrow H_{1}\left(B_{1}, \mathbb{Z}_{2}\right)\right]$; thus $B_{2}$ is a complex curve of genus $\mathbf{g}_{2}=2^{4 k-1}(k-1)+1$. Let $\Sigma \subset B_{2} \times B_{1}$ be the union of the graphs of $p$ and $\iota \circ p$. Then the homology class of $\Sigma$ is divisible by 2 . We may therefore construct a ramified double cover $M \rightarrow B_{2} \times B_{1}$ branched over $\Sigma$. The projection $f_{1}: M \rightarrow B_{1}$ is then a Kodaira fibration, with fiber $F_{1}$ of genus $2^{4 k-2}(4 k-3)+1$. The projection $f_{2}: M \rightarrow B_{2}$ is also a Kodaira fibration, with fiber $F_{2}$ of genus $4 k-2$. The signature of this doubly Kodaira-fibered complex surface is $\tau(M)=2^{4 k}(k-1)$. 
We now axiomatize those properties of these examples which we will need.

Definition Let $M$ be a complex surface equipped with two Kodaira fibrations $f_{j}: M \rightarrow B_{j}, j=1,2$. Let $\mathbf{g}_{j}$ denote the genus of $B_{j}$, and suppose that the induced map

$$
f_{1} \times f_{2}: M \rightarrow B_{1} \times B_{2}
$$

has degree $r>0$. We will then say that $\left(f_{1}, f_{2}\right)$ is a Kodaira double-fibration of $M$ if $\tau(M) \neq 0$ and

$$
\left(\mathbf{g}_{2}-1\right) \times r\left(\mathbf{g}_{1}-1\right) \text {. }
$$

In this case, $\left(M, f_{1}, f_{2}\right)$ will be called a Kodaira doubly-fibered surface.

Of course, the last hypothesis depends on the ordering of $\left(f_{1}, f_{2}\right)$, and is automatically satisfied, for fixed $r$, if $\mathbf{g}_{2} \gg \mathbf{g}_{1}$. The latter may always be arranged by simply replacing $M$ and $B_{2}$ with suitable covering spaces.

Note that $r=2$ in the explicit examples given above.

Given a Kodaira doubly-fibered surface $\left(M, f_{1}, f_{2}\right)$, let $\bar{M}$ denote $M$ equipped with the non-standard orientation, and observe that we now have two different symplectic structures on $\bar{M}$ given by

$$
\begin{aligned}
& \omega_{1}=\varepsilon \psi-f_{1}^{*} \varphi_{1} \\
& \omega_{2}=\varepsilon \psi-f_{2}^{*} \varphi_{2}
\end{aligned}
$$

for any given area forms $\varphi_{j}$ on $B_{j}$ and any sufficiently small $\varepsilon>0$.

Theorem 1 Let $\left(M, f_{1}, f_{2}\right)$ be any Kodaira doubly-fibered complex surface. Then for any self-diffeomorphism $\Phi: M \rightarrow M$, the symplectic structures $\omega_{1}$ and $\pm \Phi^{*} \omega_{2}$ are deformation inequivalent.

That is, $\omega_{1},-\omega_{1}, \Phi^{*} \omega_{2}$, and $-\Phi^{*} \omega_{2}$ are always in different path components of the closed, non-degenerate 2 -forms on $\bar{M}$. (The fact that $\omega_{1}$ and $-\omega_{1}$ are deformation inequivalent is due to a general result of Taubes [10], and holds for any symplectic 4 -manifold with $b^{+}>1$ and $c_{1} \neq 0$.)

Theorem 1 is actually a corollary of the following result:

Theorem 2 Let $\left(M, f_{1}, f_{2}\right)$ be any Kodaira doubly-fibered complex surface. Then for any self-diffeomorphism $\Phi: M \rightarrow M$,

$$
\Phi^{*}\left[c_{1}\left(\bar{M}, \omega_{2}\right)\right] \neq \pm c_{1}\left(\bar{M}, \omega_{1}\right) .
$$


Proof Because $\tau(M) \neq 0$, any self-diffeomorphism of $M$ preserves orientation. Now $M$ is a minimal complex surface of general type, and hence, for the standard 'complex' orientation of $M$, the only Seiberg-Witten basic classes [8] are $\pm c_{1}(M)$. Thus any self-diffeomorphism $\Phi$ of $M$ satisfies

$$
\Phi^{*}\left[c_{1}(M)\right]= \pm c_{1}(M) .
$$

Letting $F_{j}$ be the Poincaré dual of the fiber of $f_{j}$, and letting $\mathbf{g}_{j}$ denote the genus of $B_{j}$, we have

$$
c_{1}\left(\bar{M}, \omega_{j}\right)=c_{1}(M)+4\left(\mathbf{g}_{j}-1\right) F_{j}
$$

for $j=1,2$. The adjunction formula therefore tells us that

$$
\left[c_{1}\left(\bar{M}, \omega_{j}\right)\right] \cdot\left[c_{1}(M)\right]=(2 \chi+3 \tau)(M)-2 \chi(M)=3 \tau(M) \neq 0,
$$

where the intersection form is computed with respect to the 'complex' orientation of $M$.

If we had a diffeomorphism $\Phi: M \rightarrow M$ with $\Phi^{*}\left[c_{1}\left(\bar{M}, \omega_{2}\right)\right]= \pm c_{1}\left(\bar{M}, \omega_{1}\right)$, this computation would tell us that that

$$
\Phi^{*}\left[c_{1}(M)\right]=c_{1}(M) \Longrightarrow \Phi^{*}\left[c_{1}\left(\bar{M}, \omega_{2}\right)\right]=c_{1}\left(\bar{M}, \omega_{1}\right)
$$

and that

$$
\Phi^{*}\left[c_{1}(M)\right]=-c_{1}(M) \Longrightarrow \Phi^{*}\left[c_{1}\left(\bar{M}, \omega_{2}\right)\right]=-c_{1}\left(\bar{M}, \omega_{1}\right) .
$$

In either case, we would then have

$4\left(\mathbf{g}_{1}-1\right) F_{1}=c_{1}\left(\bar{M}, \omega_{1}\right)-c_{1}(M)= \pm \Phi^{*}\left[c_{1}\left(\bar{M}, \omega_{2}\right)-c_{1}(M)\right]= \pm 4\left(\mathbf{g}_{2}-1\right) \Phi^{*}\left(F_{2}\right)$.

On the other hand, $F_{1} \cdot F_{2}=r$, so intersecting the previous formula with $F_{2}$ yields

$$
4\left(\mathbf{g}_{1}-1\right) r=4\left(\mathbf{g}_{1}-1\right) F_{1} \cdot F_{2}=4\left(\mathbf{g}_{2}-1\right)\left[ \pm \Phi^{*}\left(F_{2}\right) \cdot F_{2}\right],
$$

and hence

$$
\left(\mathbf{g}_{2}-1\right) \mid r\left(\mathbf{g}_{1}-1\right),
$$

in contradiction to our hypotheses. The assumption that $\Phi^{*}\left[c_{1}\left(\bar{M}, \omega_{1}\right)\right]=$ $\pm c_{1}\left(\bar{M}, \omega_{2}\right)$ is therefore false, and the claim follows.

Theorem 1 is now an immediate consequence, since the first Chern class of a symplectic structure is deformation-invariant.

Acknowledgment This work was supported in part by NSF grant DMS0072591. 


\section{References}

[1] M F Atiyah, The signature of fibre-bundles, from "Global Analysis (Papers in honor of K Kodaira)", Univ. Tokyo Press, Tokyo (1969) 73-84

[2] W Barth C Peters, A V de Ven, Compact complex surfaces, Springer-Verlag (1984)

[3] K Kodaira, A certain type of irregular algebraic surfaces, J. Analyse Math. 19 (1967) 207-215

[4] D Kotschick, Signatures, monopoles and mapping class groups, Math. Res. Lett. 5 (1998) 227-234

[5] N C Leung, Seiberg-Witten invariants and uniformizations, Math. Ann. 306 (1996) 31-46

[6] D McDuff, D Salamon, Introduction to symplectic topology, Oxford Science Publications, The Clarendon Press and Oxford University Press, New York (1995)

[7] C T McMullen, C H Taubes, 4-manifolds with inequivalent symplectic forms and 3-manifolds with inequivalent fibrations, Math. Res. Lett. 6 (1999) 681-696

[8] J Morgan, The Seiberg-Witten equations and applications to the topology of smooth four-manifolds, vol. 44 of Mathematical Notes, Princeton University Press (1996)

[9] J Petean, Indefinite Kähler-Einstein metrics on compact complex surfaces, Comm. Math. Phys. 189 (1997) 227-235

[10] C H Taubes, More constraints on symplectic forms from Seiberg-Witten invariants, Math. Res. Lett. 2 (1995) 9-14 